\title{
Mapeando la enseñanza de biliteracidad en contextos indígenas: Desde la timidez hacia la voz ${ }^{1}$
}

\author{
Nancy H. Hornberger \\ University of Pennsylvania \\ nancyh@upenn.edu \\ Frances Kvietok Dueñas \\ Universidad Peruana Cayetano Heredia \\ frances.kvietok@upch.pe
}

Recibido: 12-11-2019

Aprobado: 22-11-2019

1. Este trabajo ha sido publicado originalmente en inglés como Hornberger, Nancy H., Kvietok Dueñas, Frances (2019). Mapping biliteracy teaching in Indigenous contexts: From student shyness to student voice. Anthropology and Education Quarterly, 50(1), 6-25. De manera excepcional, publicamos la traducción de este artículo por su aporte a la comunidad académica y decisores de política en el marco de la implementación de la Política Sectorial de Educación Intercultural y Educación Intercultural Bilingüe (2016) y la Política de Atención Educativa para la Población en Ámbitos Rurales (2018). 


\title{
Resumen
}

Basadas en una experiencia de monitoreo etnográfico con maestros bilingües interculturales kichwa en la Amazonía peruana, proponemos el uso del monitoreo etnográfico como método y las continuidades de la biliteracidad como heurística para el mapeo de la enseñanza de la biliteracidad en contextos indígenas de bilingüismo. A través de nuestro mapeo, revelamos tensiones en la enseñanza de lenguas mayoritarias en contextos poscoloniales indígenas, cuestionamos la ideología de la timidez del niño indígena y proponemos pedagogías que apoyen el florecimiento de su voz en la educación bilingüe.

Palabras clave: monitoreo etnográfico, educación bilingüe, enseñanza del castellano como segunda lengua, pedagogías de la voz

\section{Mapping biliteracy teaching in Indigenous contexts: From student shyness to student voice}

\begin{abstract}
Drawing on an ethnographic monitoring engagement with Kichwa intercultural bilingual educators in the Peruvian Amazon, we argue for ethnographic monitoring as a method and the continua of biliteracy as a heuristic for mapping biliteracy teaching in Indigenous contexts of bilingualism. Through our mapping, we uncover tensions in the teaching of majoritized languages in Indigenous contexts of postcoloniality, challenge constructs of student shyness, and propose pedagogies to support the flourishing of student voice in bilingual education.

Keywords: ethnographic monitoring, bilingual education, Spanish as second language, pedagogies of voice
\end{abstract}




\section{Introducción}

Pedro $^{2}$, un maestro bilingüe kichwa-castellano, empieza su lección de castellano con estas palabras: "Aquí hablamos el kichwa cien por ciento; el problema está en castellano" (NC ${ }^{3}$ 2013-06-26). Su colega Eric, al término de su clase de castellano, comenta a sus alumnos: "nuestra lengüita está todavía dura" (NC 2013-06-20). Pedro y Eric son nativos de la región del Alto Napo en la Amazonía peruana, sede del pueblo kichwa (napuruna) y de una red de seis escuelas primarias bilingües interculturales. Ellos se identifican como kichwas y maestros bilingües en castellano y kichwa, una variedad de quechua hablada en el Alto Napo del Perú. Ambos se preocupan sinceramente en apoyar el aprendizaje bilingüe kichwa-castellano de sus alumnos por motivos políticos y educativos.

Al mismo tiempo, ambos señalan la timidez de los niños como un obstáculo primordial para enseñarles el castellano y responden a esta preocupación a través de sus prácticas pedagógicas. Pedro menciona: "la dificultad [...] que los niños tienen, o sea mucho tienen vergüenza de expresar, tienen timideces, [se sienten] incomodos allí” (E 2013-06-28) . Otros maestros del Alto Napo usaron como una expresión común el "miedo de hablar" para referirse a la relación que tienen los niños con el castellano. La maestra Renata, colega de Pedro y Eric, explicó: "[mis alumnos son] como el mudo nomás [...] venga para acá, ya se mueve, vaya para allá, ya se mueve [...] pero no pronuncia; tiene que pronunciar" (E 2013-07-10). Así, enfatiza su preocupación con alumnos que entienden, pero no hablan el castellano.

Un reto enorme en los sistemas educativos latinoamericanos sigue siendo la apertura a espacios para múltiples lenguas e identidades dentro de sociedades que desde hace siglos se adhieren a la ideología una nación-una lengua, y que promueven discursos de homogeneidad lingüística y cultural. Este reto continúa vigente en la Amazonía peruana, donde los sistemas educativos han prohibido y limitado el rol y uso de lenguas y conocimientos locales, privilegiando en cambio la literacidad en castellano (Aikman, 1999). Diversos investigadores también subrayan que la enseñanza y el aprendizaje del castellano es una demanda permanente de comunidades y organizaciones indígenas, mientras que la oferta de formación profesional que apoya a estas pedagogías perdura como una preocupación (Aikman, 1999; Jung y López, 2003).

En este artículo, nos enfocamos en las experiencias de aula de maestros kichwas del castellano como segunda lengua en contextos poscoloniales de

2. Todos los nombres son seudónimos.

3. "E" se refiere a las entrevistas; "NC", a las notas de campo; y "A", a grabaciones de audio.

4. Todas las entrevistas se realizaron en castellano. En todas las transcripciones, se han respetado las prácticas lingüísticas de los entrevistados. 
bilingüismo indígena en América Latina ${ }^{5}$. Sobre la base de estos casos ilustrativos, proponemos el monitoreo etnográfico (Hymes, 1980) como método y las continuidades de la biliteracidad (Hornberger, 1989) como heurística para mapear y reencaminar la enseñanza de lenguas mayoritarias ${ }^{6}$ en contextos de biliteracidad hacia pedagogías de la voz.

\section{Marco conceptual y metodológico}

"Hymes propuso no solo una visión sino también un conjunto de formas de realizar etnografía en la educación-desde el monitoreo etnográfico y la etnografía de la comunicación a la etnopoética de la narrativa oral y la etnografía de la política lingüística" y abogó por "la etnografía como teoría y perspectiva, como descripción y análisis de la complejidad y el enredo de la actividad social, [y] como antihegemónica y democrática, accesible al experto y al principiante"7 (Hornberger, 2009, p. 355). En particular, Hymes propuso que el monitoreo etnográfico de programas educativos apoya a los investigadores a (i) describir las prácticas comunicativas locales existentes, (ii) analizar e interpretar los patrones y significados de la implementación del programa, y (iii) evaluar los efectos y consecuencias políticas del programa con miras a promover logros escolares y cuestionar nociones aceptadas de la desigualdad lingüística (Hymes, 1980; Hornberger, 2014). Hymes urgió a los monitores a aprovechar el potencial democrático de la etnografía, la cual provee de oportunidades para que los actores locales sean participantes en el monitoreo de los programas de educación bilingüe, dado que "ellos son los que tienen el mejor entendimiento del funcionamiento del programa" (1980, p. 115), y son quienes deciden si hacer uso de los hallazgos etnográficos y de qué manera.

Refiriéndose al informe de un proyecto de monitoreo etnográfico en escuelas de la ciudad de Filadelfia a lo largo de tres años (Hymes, 1981), Van der Aa y Blommaert ponen énfasis en "la colaboración intensiva y genuina [...] que reside en el corazón del monitoreo etnográfico" (2011, p. 324). En un contexto poscolonial como el de Alto Napo, esta colaboración no se trata de una mera cortesía, sino de un método respetuoso y orientado hacia la praxis. De Korne y Hornberger abordan este tema, subrayando desde sus experiencias de monitoreo etnográfico que "los etnógrafos no se limitan solo a describir la realidad social [...] sino pueden también intentar monitorear cambios tanto

5. Con los términos poscolonialidad y colonialidad continua, nos referimos tanto al pasado colonial que es parte del legado de estados-naciones (como el Perú) como también a formas de explotación y opresión continuas (Quijano, 2000).

6. Usamos los términos "mayoritarias" y "mayorizadas" para referirnos a las lenguas dominantes, aquellas que se han construido como socialmente dominantes, mientras que, con el término "minorizadas", aludimos a las relaciones de poder que posicionan a lenguas con menor estatus, independientemente de la cantidad de hablantes. 
positivos como negativos", apoyando a lograr los objetivos locales (2017, pp. 247-8).

Aquí introducimos el mapeo a través de las continuidades de la biliteracidad como una herramienta de apoyo para investigadores con el fin de alcanzar las metas críticas y colaborativas del monitoreo etnográfico. El marco de las continuidades de la biliteracidad ofrece una heurística para situar la investigación, la enseñanza, y la formulación de la política y la planificación lingüística en contextos multilingües. Con premisas en décadas de investigación sobre el bilingüismo y la literacidad, la heurística de las continuidades señala que categorías oposicionales como primera y segunda lengua, monolingüe-bilingüe, $u$ oralidad-literacidad son en realidad continuidades fluidas y dinámicas del uso de lengua y literacidad, dimensiones interrelacionadas de repertorios comunicativos complejos y fluidos (Gumperz, 1964). En estos espacios dinámicos; cambiantes; y, a veces, disputados a lo largo y a través de las continuidades, ocurre el uso y aprendizaje de la biliteracidad (Hornberger, 1989, 2003).

La biliteracidad se trata del uso tanto oral como escrito de la lengua, entendida como "cada una y todas las instancias en las cuales ocurre comunicación en dos (o más) lenguas en, o en torno a, la escritura" (Hornberger, 1990, p. 213). Esta definición pone el énfasis en la interacción oral y escrita, a diferencia de otras definiciones que circunscriben a la biliteracidad a (el dominio de) la lectura (y escritura) en dos lenguas. De igual manera, esta definición señala "instancias" de biliteracidad, que incluyen tanto a los actores biletrados, como a los eventos y prácticas en que interactúan, y a los espacios y mundos donde lo hacen.

El modelo consiste en cuatro espacios tridimensionales y encajados, compuestos de las continuidades que caracterizan contextos, medios, contenidos y desarrollo de la biliteracidad. Específicamente, el modelo describe el desarrollo de la biliteracidad a través de las continuidades comprensión-expresión, lengua oral-escrita, y primera-segunda lengua (tercera, cuarta, etc.) por medio de dos (o más) lenguas. Las estructuras de dichas lenguas varían en su relación entre sí desde lo disimilar a lo similar y sus ortografías se distribuyen entre lo más divergente a lo más convergente. Además, estas lenguas se adquieren en procesos que fluctúan desde lo simultáneo a lo sucesivo, en contextos que comprenden niveles o escalas desde lo micro hasta lo macro, y se caracterizan por variadas combinaciones de oralidad-literacidad y multilingüismo-monolingüismo. Por último, los contenidos de estas lenguas abarcan experiencias y perspectivas desde lo minorizado a lo mayorizado, estilos y géneros desde lo vernacular a lo literario, y textos que varían desde lo contextualizado a lo descontextualizado.

Los conceptos de "continuidades" y "biliteracidad" son los fundamentos del esquema (ver Figura 1), que postula que cuanto más los contextos de aprendizaje y uso de la lengua y la literacidad propicien que los individuos multilingües aprovechen cada una de las continuidades en su totalidad, tantas más son las posibilidades para el desarrollo y expresión de su biliteracidad. Para ese fin, es necesario contrarrestar las prácticas lingüísticas y letradas tradicionales y 
hegemónicas en la educación - prácticas monolingües, escritas y descontextualizadas - abriendo en su lugar espacios implementacionales e ideológicos para prácticas y voces fluidas, multilingües, orales y contextualizadas al nivel local (Hornberger, 2002).

Como herramienta para lograr este propósito, proponemos el mapeo de la enseñanza del castellano en el Alto Napo a través de las continuidades de la biliteracidad. Dado su potencial crítico y predictivo, el marco de las continuidades de la biliteracidad se adecúa a los objetivos del monitoreo etnográfico, enfocados en la mejora de aprendizajes y en el cuestionamiento de nociones aceptadas de la desigualdad lingüística. Asimismo, intentaremos mapear cómo se pueden reencaminar las prácticas de la biliteracidad en castellano para abrir espacios donde las voces de los alumnos puedan florecer.

\section{Figura 1}

Las continuidades de la biliteracidad

Relaciones de poder en las continuidades de biliteracidad

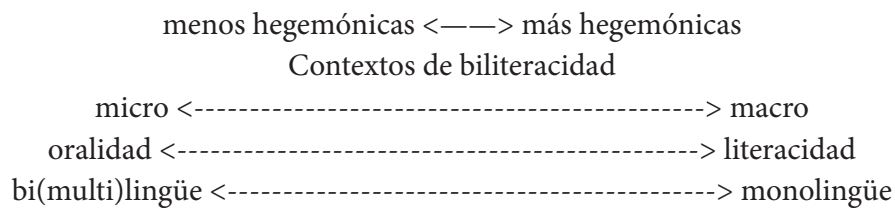

Desarrollo de la biliteracidad

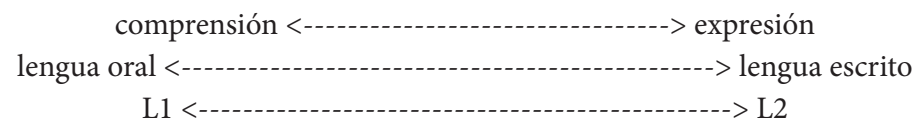

Contenidos de la biliteracidad

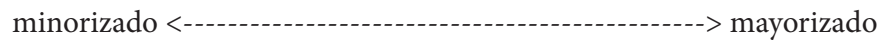

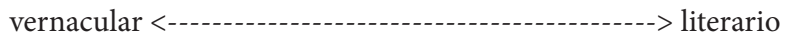

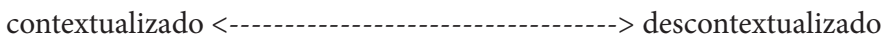

Medios de la biliteracidad

adquisición simultanea <-------------------> adquisición sucesiva

estructuras disimilares $<--------------------------->$ estructuras similares

ortografías divergentes $<----------------------->$ ortografías convergentes

Fuente: Reimpreso de Hornberger y Skilton-Sylvester $(2000)^{8}$.

104 8. La imagen de Hornberger y Skilton-Sylvester (2000) se ha reproducido con permiso de la editorial. 
Para abordar la noción de voz, nos inspiramos en Ruiz (1997), quien subrayó la clara distinción entre incluir al lenguaje del alumno en la educación y apoyar la voz de los aprendices. Para Ruiz, la lengua es algo general, una abstracción que existe aún cuando se la suprime, mientras que la voz es "particular y concreta". El autor señala que tener voz implica "no solamente que uno puede decir algo, sino que es escuchado (es decir, que sus palabras tienen estatus, influencia)" (1997, p. 321). Claro está que ni las escuelas ni los maestros pueden brindarles voz a los individuos; sin embargo, sugerimos que los educadores sí pueden transformar ideologías de timidez estudiantil indígena en condiciones para la voz y, a cambio, construir lo que llamamos pedagogías de la voz. En este artículo, consideramos la importancia de crear condiciones para que florezca la voz estudiantil indígena (Hornberger, 2006) en la enseñanza y aprendizaje de lenguas dominantes, la cual debería llevarse a cabo de la mano con el mantenimiento y la revitalización de las lenguas indígenas.

\section{El Alto Napo: Escenario, participantes y métodos}

Las desigualdades educativas que afrontan los pueblos originarios se encuentran enmarcadas dentro de largas historias de colonialidad continua y de trayectorias de luchas constantes. El Alto Napo peruano es una región a 2 días de viaje de la ciudad Iquitos, cuyas décadas de dominación y esclavitud iniciaron en 1538, con la llegada de la invasión española a la zona y la instauración del sistema de la encomienda. Desde finales del siglo XIX, una economía feudal y extractiva controlada por caucheros, patrones y regatones mantuvo estas condiciones coloniales y opresivas (Mercier, 1983, p. 30). En la actualidad, los napuruna sostienen relaciones tensas con la industria petrolera, minera y maderera, cuyas actividades conllevan la contaminación del territorio kichwa al lado de promesas incumplidas de desarrollo (Aidesep, 2013).

Desde fines del siglo XX, las comunidades kichwa comenzaron a crear espacios locales para transformar los discursos y las prácticas educativas que favorecían al monolingüismo en castellano. En un contexto de surgimiento de políticas de educación bilingüe en los años 70 , misioneros católicos y maestros kichwas crearon el Pebian (Programa de Educación Bilingüe e Intercultural en el Alto Napo), y desarrollaron una educación intercultural y bilingüe para los niños kichwa, lo cual representaba un esfuerzo pionero desde las bases (Ashanga Jota et al., 1990). Aunque compartía similitudes con otros modelos de educación bilingüe alrededor del mundo en esa época, el Pebian transcendió el discurso dominante de integración nacional reflejado en las políticas educativas y promovió "un modelo de educación orientado a la justicia social, enfocado en el desarrollo de relaciones interculturales y la transformación [social]" (Kvietok Dueñas, 2015, p. 30).

Más recientemente, dentro de los espacios promovidos por la Política de Educación Intercultural y de Educación Bilingüe Intercultural, promulgada por el Gobierno peruano en 1991, varias escuelas del Alto Napo han renovado 
esfuerzos para implementar un modelo de educación intercultural y bilingüe. Desde el 2008, con el apoyo del renombrado programa de formación docente Formabiap (Programa de Formación de Maestros Bilingües de la Amazonía Peruana), la red Cebes (Comunidades y Escuelas para el Bien Estar) ha promovido la relación escuela-comunidad para implementar un modelo educativo desde y para las comunidades indígenas del Alto Napo. La red Cebes promueve el buen vivir a través de una educación que afirme la herencia cultural y la identidad de los pueblos originarios, y les permita interactuar en otros contextos de manera intercultural (Medina, 2009). La orientación intercultural de Cebes se evidencia, por ejemplo, en la participación de padres y madres de familia en la elaboración de materiales educativos para sus hijos y en la importancia del calendario comunal agrofestivo en la planificación del currículo.

Las escuelas del Alto Napo implementan un modelo de educación bilingüe de mantenimiento, cuyo objetivo es desarrollar literacidad en kichwa en los primeros grados introduciendo la literacidad en castellano del tercer grado de primaria en adelante con miras a que ambos idiomas se conviertan en medios y objetos de aprendizaje. Para el quinto y sexto grado de primaria, se busca que el castellano se convierta en el medio de enseñanza para al menos la mitad de la jornada escolar. A través de una capacitación continua, los acompañantes de la red Cebes y los docentes trabajan juntos para implementar una EIB. En la práctica, la implementación de este modelo varía: mientras que algunas escuelas siguen los objetivos del modelo, en otras, no todos los docentes trabajan la EIB.

En una evaluación inicial de las actividades de la red que abarcó a escuelas del presente estudio, se identificaron mejoras en la planificación curricular con un enfoque intercultural, en el rendimiento académico en diversas áreas y grados, y en el vínculo entre la escuela y la comunidad (Trapnell, 2010). No obstante, se identificó la persistencia de diversas dificultades influenciadas por el número limitado de docentes bilingües certificados, la alta rotación de docentes, la falta de materiales educativos bilingües y la falta de apoyo político para la EIB.

En relación con las actividades de monitoreo que describiremos, destacan los desafíos relacionados con la enseñanza y el aprendizaje del castellano. Con el apoyo del acompañante de Cebes, las escuelas del Alto Napo comenzaron a implementar sesiones diarias de treinta minutos de duración, desde el primer grado, con un enfoque en el desarrollo de la expresión oral. Se buscaba que los maestros utilizaran y modelaran el castellano oral cuanto más se pudiera para así fomentar la comprensión oral y las habilidades productivas en los alumnos desde temprana edad. No obstante, usaban el kichwa en ciertos momentos para explicar actividades o monitorear el comportamiento de los estudiantes. No se sancionaba el uso del kichwa por parte de los alumnos, aunque tampoco se fomentaba.

Este artículo se basa en datos recolectados durante el monitoreo etnográfico realizado por Kvietok Dueñas en 6 escuelas EIB de nivel primario 106 I pertenecientes a la red Cebes, llevado a cabo por 2 meses en el 2013. El moni- 
toreo etnográfico se realizó en seis comunidades kichwas situadas a lo largo del Río Napo y sus afluentes, ubicadas desde quince minutos a una hora de viaje entre sí por medio de un pequeño bote a motor. En conjunto, las 6 escuelas reunían a un total de 17 maestros ( 2 mujeres), con una experiencia docente de 3 a 32 años. La composición de las escuelas era diversa, tanto en relación con el número de docentes (1-6) como con el número de alumnos (30-168) y su organización (multigrado, unidocente y polidocente). Los docentes eran bilingües, se identificaban como kichwa, y vivían en las comunidades donde laboraban. Si bien la mayoría había crecido en comunidades del Alto Napo, la mayoría no enseñaba en sus comunidades, aunque todos hablaban la misma variedad de kichwa que sus alumnos. Su formación docente incluía estudios superiores y diversos tipos de capacitaciones. Solo dos maestros habían realizado estudios de formación docente en un programa de educación bilingüe, y la mayoría había aprendido sobre la educación bilingüe y la enseñanza de segundas lenguas a través de diversas capacitaciones, como la capacitación Cebes. El acompañante de la red Cebes se había percatado de que, muchas veces, los maestros encontraban dificultoso hacer uso de métodos de enseñanza bilingüe que habían aprendido durante su formación y, más bien, solían hacer uso de estrategias que reflejaban la manera en que ellos mismos habían aprendido.

Las sesiones diarias de castellano oral habían comenzado un año antes de la visita de Kvietok Dueñas y fueron el foco de su monitoreo etnográfico. Antes de su llegada a la zona, miembros de la red Cebes compartieron algunas de las dificultades de implementación observadas, incluidos la distribución irregular de tiempos, la falta de métodos y materiales pertinentes, y el hecho de que no se aplicaba la separación de grupos según niveles de castellano recomendada a los docentes. Kvietok Dueñas se incorporó a las escuelas en el rol de capacitadora de castellano como segunda lengua en función de las necesidades expresadas por el equipo local.

Además de facilitar reuniones de capacitación, modelar sesiones de castellano como L2, y participar en sesiones de planificación junto con los docentes, Kvietok Dueñas realizó observación participante en las seis escuelas (doce clases esparcidas a través de todos los grados) y entrevistas semiestructuradas con cinco docentes (tres hombres y dos mujeres). Todas estas actividades formaron parte del monitoreo etnográfico. Las observaciones se enfocaron en las clases de castellano, aunque también se observaron clases en kichwa para desarrollar una mejor comprensión del contexto educativo. Conviviendo con familias en varias de las comunidades visitadas durante el periodo de monitoreo, Kvietok Dueñas participó en diversas reuniones, eventos escolares y comunitarios.

Durante el monitoreo etnográfico, Kvietok Dueñas buscó apartarse de una visión homogénea de los actores educativos y aproximarse hacia una concepción más íntegra de los diversos miembros que componían la comunidad con la que colaboraba. Entre los actores locales, se encontraban los directores, los docentes, el acompañante Cebes y los alumnos, y, en una escala más amplia, los 
docentes y los directivos de Formabiap. El proceso de monitoreo etnográfico también involucró una apertura para considerar los diferentes roles, vínculos y maneras de efectuar los cambios que surgieran. Por ejemplo, fue necesario adaptarse a las diferentes maneras en que diversos actores preferían recibir retroalimentación. Mientras que miembros de Formabiap mostraban una preferencia por los reportes orales y escritos, los docentes preferían las conversaciones $\mathrm{y}$, frecuentemente, solicitaban demostraciones y el modelado de didácticas en sus aulas. Otro ejemplo se reflejó en la necesidad de moldear el monitoreo alrededor de diferencias significativas entre maestros, entre las cuales destacaron los roles de género. Debido a que las maestras dedicaban gran parte de su tiempo después de la escuela a las labores domésticas, las actividades de monitoreo se adaptaron a sus rutinas, por lo cual muchas de las conversaciones con estas docentes se llevaron a cabo mientras se apoyaba en la realización de estas labores. Los maestros, por su parte, se dedicaban a la caza y la pesca antes y después de la jornada escolar, por lo cual las entrevistas con ellos tuvieron que ser reagendadas en múltiples ocasiones. En conjunto, estos ejemplos revelan la necesidad de mantener una mirada compleja a lo que significa colaborar con la comunidad local en el monitoreo.

\section{Hallazgos: Mapeando la enseñanza del castellano en el Alto Napo}

En esta sección, presentaremos nuestros hallazgos en tres partes. Primero, contextualizaremos la enseñanza de la biliteracidad en castellano en el Alto Napo; luego, consideraremos las prácticas comunicativas en el aula del castellano como L2; y, finalmente, nos enfocaremos en los esfuerzos colaborativos entre Kvietok Dueñas y los maestros para reencaminar la enseñanza del castellano hacia pedagogías de la voz. En cada parte, seguimos los tres ciclos etnográficos delineados por Hymes. Primero, describimos los contextos, las prácticas y los esfuerzos colaborativos para, en segundo lugar, analizar e interpretar los patrones y significados emergentes para los participantes. Finalmente, realizamos una evaluación de los efectos y las consecuencias políticas. El mapeo en tres escalas acompañado por los tres ciclos etnográficos refleja el carácter iterativo de las actividades del monitoreo, las cuales emergían y se apoyaban entre sí.

\section{Escala 1: Ecologías locales de desigualdad lingüística y la enseñanza del castellano}

Empezamos con una mirada a la ecología local de lenguaje y su implicancia educativa en el Alto Napo.

\section{Describiendo, analizando e interpretando las ecologías lingüisticas}

Las ecologías lingüísticas del Alto Napo se caracterizan por un desbalance he108 I gemónico del repertorio de prácticas comunicativas monolingües-multilingües 
y orales-letradas. Tanto para adultos como para niños, el kichwa sigue siendo la lengua del hogar y de mayor uso en las actividades cotidianas, como el cultivo, la caza, la pesca, las reuniones sociales y los trabajos minga. Al mismo tiempo, se demanda y valora el castellano oral y escrito. El castellano es mediador del acceso a servicios del gobierno, donde los trabajadores no hablan el kichwa (centros de salud, servicios sociales). Además, esta lengua facilita la participación en asambleas comunales cuando hay presencia de foráneos, y habilita transacciones con comerciantes de tiendas locales o del río que no son kichwas. En general, los niños tienen su primer encuentro con el castellano al entrar a la escuela. A diferencia de las madres, algunos padres de familia lo entienden y hablan en cierta medida. Esta situación responde a que los hombres tienen más oportunidades de seguir estudios secundarios en castellano y salir de las comunidades por razones de empleo. Afuera de las comunidades, el castellano media el acceso al empleo y al estudio, y estas actividades ofrecen mayor oportunidad para el aprendizaje del castellano.

A través del monitoreo, era evidente que los contextos para el uso del castellano tanto dentro como afuera de las comunidades son situaciones de desigualdad en que los kichwa-hablantes no disponen del capital lingüístico (o de otro capital) para interactuar en términos de igualdad con los castellanohablantes. En una asamblea comunal donde Kvietok Dueñas estuvo presente, un empleado del gobierno buscaba formar un comité para supervisar la instalación de la electricidad. Dirigiéndose al grupo en castellano, explicó que un diploma de la secundaria era requisito para incorporarse al comité y expresó que sería bueno que una mujer que escriba bien ocupe el rol de tesorera del comité (NC 2013-07-14), aparentemente, con la idea de que una mujer sería más confiable cuando se trata de fondos monetarios. En esta asamblea comunal facilitada en castellano, no solo se privilegiaron las habilidades productivas orales en castellano y se limitó la participación de los kichwa-hablantes, sino también se posicionó la literacidad escolar en castellano como requisito para participar en el liderazgo de la comunidad kichwa. Aún más conmovedor es que las mujeres, a quienes supuestamente se animaba a participar, fueron simultáneamente excluidas por su escolaridad limitada y su menor conocimiento del castellano. Esta situación demuestra claramente un conflicto entre las expectativas para un liderazgo femenino y la desigualdad de género endémica en el acceso al castellano.

La valoración social del castellano subyace también en los discursos de superación: la idea de que los niños superen a sus padres en el adelanto socioeconómico. Los padres hablaban del aprendizaje del castellano como una vía por la cual la juventud podría superarse, ya sea porque les facilita su entrada a la secundaria o porque abre acceso a empleo fuera de las comunidades. A la vez, recalcaban con frecuencia las habilidades limitadas de sus hijos para hablar castellano. Una madre comentó que, para ella, era notable que muchos niños y niñas, cuando se les pregunta algo en castellano, simplemente miran y sonríen, sin responder. Mencionó que su deseo era que los niños y las niñas 
hablaran ambos idiomas con confianza, y que era responsabilidad de la escuela cumplir con esta tarea (NC 2013-06-23). Es importante notar, también, que las metas de los padres para la educación y el aprendizaje del castellano se enmarcaban dentro de metas más amplias del apoyo al bienestar de las comunidades kichwas. Como explicó un apu (líder) de la comunidad, así como es importante que los jóvenes salgan de las comunidades para estudio y empleo, también es necesario que vuelvan y apoyen a las comunidades y a la organización política napuruna (NC 2013-07-11).

\section{Evaluando efectos y consecuencias para los maestros}

Los maestros eran sumamente conscientes de los contextos desiguales del uso del castellano y de la biliteracidad, debido a que la mayoría de ellos habían crecido en el Alto Napo. El maestro Daniel, por ejemplo, consideraba que la enseñanza del castellano a sus niños es "como un defensa personal para ellos" (E 2013-07-04) en contextos que predominantemente favorecen las habilidades orales y letradas en castellano. Enseña castellano a sus estudiantes para que puedan valerse por sí mismos en situaciones en que los de afuera tradicionalmente se aprovechan de los napuruna, engañándolos en transacciones - como la venta de madera, cerdos, y otros bienes materiales- por el mero hecho de "habla[r] mejor castellano". Otros maestros enfatizaban el rol importante de la literacidad en castellano en el liderazgo kichwa, como, por ejemplo, en la Asociación de Padres de Familia (APAFA), o como medio de interacción local con oficiales del gobierno u otros actores de la sociedad civil desde afuera (E 2013-07-10), o para el estudio o empleo en ciudades (E 2013-07-05).

Los discursos de los maestros sobre el castellano como herramienta de autodefensa y liderazgo van acompañados de objetivos para la enseñanza y el aprendizaje del castellano específicos para cada grado, objetivos que compartían un énfasis en el desarrollo de habilidades de producción oral. El maestro Javier articuló su deseo de que sus estudiantes del primer grado "se expresen, que hablen, que participen en castellano hablando" (E 2013-07-16) y el maestro Pedro señaló que deseaba que sus estudiantes del cuarto grado "hablen castellano sin tener miedo" (E 2013-06-28). Los maestros, generalmente, consideraban sus clases de expresión oral como espacios para superar la timidez de los niños y sus habilidades limitadas en castellano. De esta manera, indexan una visión de los estudiantes como aprendices del castellano con dificultades permanentes, dada la poca presencia del castellano en sus hogares y en los grados inferiores. Por ejemplo, la maestra Renata explicó que la mayoría de los estudiantes tenía su primer y mayor encuentro con el castellano en el aula, "Porque más en la casa todo es su idioma, kichwa [...] vienen a la escuela y escuchan castellano, pero medio raro para ellos" (E 2013-07-10). El maestro Pedro estaba de acuerdo con esto, señalando que aun si los padres entienden el castellano, no lo hablan, lo cual no ofrece un apoyo para que sus hijos lo apren110 I dan. Del mismo modo, los maestros notaban que el hecho de que en los grados 
inferiores no se haya trabajado contenidos contextualizados ni relevantes en castellano tampoco apoyaba a que los niños superen su timidez para hablar castellano (E 2013-07-10; 2013-07-04).

A menudo, los maestros indexaban esta visión de aprendices del castellano con dificultades permanentes situados en una ecología de desigualdad lingüística en el hogar y el colegio. Para el maestro Pedro, algunos estudiantes eran más "quedados" que otros y no participaban en clase debido a dificultades para hablar el castellano. Por otro lado, los estudiantes que sí participaban eran "un poco más despiertos [...] son activos, y al toque [...] escuchando nomás aprenden cosas" (E 2013-06-28). Para el maestro Daniel, aprender a leer en castellano dependía del esfuerzo individual del estudiante: "Ya pues depende de ti, yo mis ojos no te voy a dar a ti, mi boca ni mi vocación tampoco, sino tú preocúpate, practica [...]" (E 2013-07-04). Los comentarios de los maestros recurrentemente reflejaban la ideología de que los estudiantes debían luchar para aprender el castellano, y que sus dificultades para adquirir y usar sus repertorios emergentes de lengua y literacidad recaían principalmente en su falta de participación y motivación.

Los maestros, también, invocaban esta concepción del estudiante como aprendiz del castellano con dificultades permanentes cuando recordaban sus propias experiencias de aprendizaje del castellano durante sus estudios:

Maestro Pedro: [en la secundaria] tuve problemas en entender algunas cosas, pero así he logrado a hablar el castellano [...] cuando salía a exponer algunos trabajos en secundaria allí se aprende bastante [...] [en] la educación superior [...] habían como cuatro pueblos que poco hablaban el castellano [...] habían unas dificultades en el castellano y así hemos logrado aprender. (E 2013-06-28)

En la experiencia del maestro Pedro, aprender el castellano significa aprender a hablar el castellano, lo cual se logra mediante presentaciones orales e interacciones con otros, sobretodo, otros que no hablan kichwa. Muy poco de su aprendizaje se lo atribuía a un enfoque educativo o una pedagogía del castellano como L2. El maestro Pedro afirma haber aprendido el castellano mediante la lucha y la práctica, y no por haber sido enseñado este idioma.

Mediante nuestra exploración de la ecología local del lenguaje en el Alto Napo, vemos, entonces, que las prácticas comunicativas y educativas alrededor de la enseñanza y aprendizaje del castellano se encuentran enmarcadas dentro de contextos de colonialidad y resistencia, y por las experiencias e ideologías de los maestros. Además, dichas prácticas están encuadradas dentro de metas más amplias de mayor representación política y bienestar socioeconómico para las comunidades kichwa. A continuación, nuestro monitoreo ilumina cómo los maestros en estos contextos dan sentido a las continuidades de la biliteracidad al trasformar sus objetivos pedagógicos en prácticas del aula. 


\section{Escala 2: prácticas comunicativas del aula}

Comenzamos por describir, analizar e interpretar dos viñetas etnográficas representativas de las prácticas de aula observadas en el Alto Napo. El maestro Pedro, quien enseñaba el cuarto grado, había comenzado su carrera como docente cinco años atrás, tras graduarse del Programa Formabiap. El maestro Eric, quien enseñaba una clase de tercero y cuarto grado, había comenzado a enseñar cuatro años atrás y no había asistido a un programa de formación bilingüe.

\section{Describiendo una sesión del maestro Pedro}

Viñeta 1: Haciendo una canoa

Los alumnos se sientan en un semicírculo, mirando hacia la pizarra. El maestro Pedro comienza comentando al grupo "aquí hablamos el kichwa cien por ciento, el problema está en castellano". Ha preparado un afiche que muestra a un hombre haciendo una canoa, el cual cuelga en la pizarra (Figura 2). Su plan es que los alumnos describan las actividades que observan en el afiche de manera oral y en castellano. Pregunta al grupo cómo elaboran sus padres una canoa y, durante los siguientes 45 minutos, la interacción se lleva a cabo de la siguiente manera:

1 MP (Maestro Pedro): ...qué cosa primero se debe hacer?

O sea se debe?

2 A (Alumno/a): (bajo) pedir

3 MP: pedir al?

4 A: dueño

5 MP: dueño

6 A: (voz baja) del cedro

7 MP: del?

8 A: cedro

9 MP: árbol, cedro, ya? No vas a cortar por gusto así cortando cortando porque si cortamos así se va rom-?

10 As (alumnos): -per

11 MP: -per, al momento que caiga la tierra se va?

12 A: [romper]

13 MP: [rajarse]

Los alumnos responden a las preguntas del maestro, a veces, de manera individual y en castellano; y, otras veces, en coro de manera conjunta. El maestro Pedro, también, realiza algunos juegos con la clase. En uno de estos juegos, los alumnos se sientan formando un círculo y cada uno elige una fruta. Uno de los alumnos se para en el centro del círculo y dice la 
frase "El pájaro pícaro loco picó un/una [nombre de una fruta]". El alumno que eligió la fruta mencionada ahora lidera el juego. Después de los juegos, el maestro Pedro pide al grupo que trabajen en parejas, y escriban una oración o un párrafo sobre lo que observaron en el afiche, además de incluir un dibujo (NC y A 2013-06-26).

Figura 2

Afiche usado por el maestro Pedro

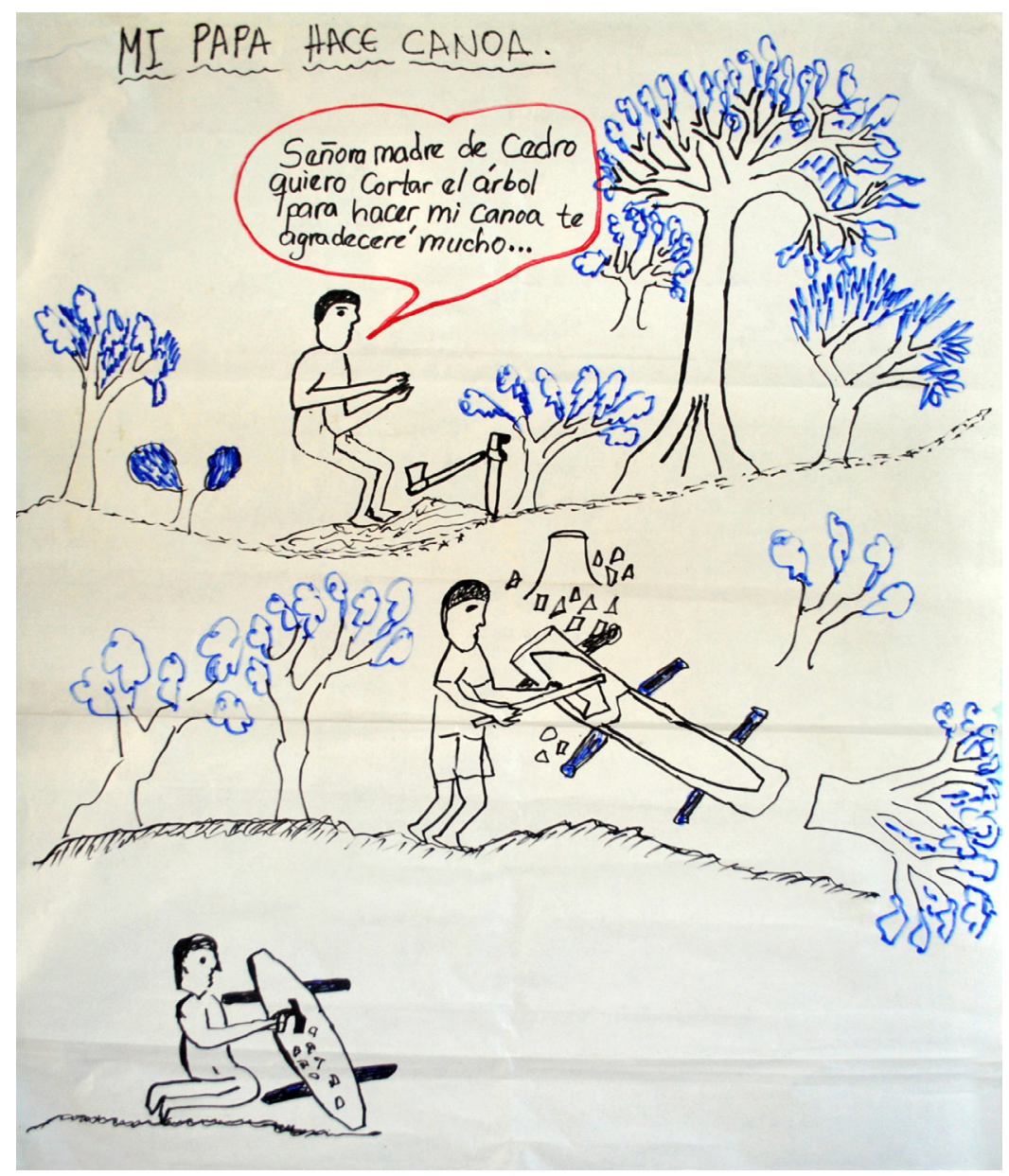

Tomando en cuenta las maneras en las que la competencia comunicativa en castellano es desarrollada, la primera actividad, que consiste en la tarea de narrar los pasos para elaborar una canoa, busca apoyar a los alumnos en el desarrollo de preciadas habilidades productivas y orales en castellano. La 
actividad también incorpora los conocimientos previos de los estudiantes, debido a que el maestro considera las prácticas discursivas locales utilizadas en esta tarea, las maneras de cortar un árbol (líneas 1 y 9), y los diferentes usos que tienen las canoas (no mostrado en la viñeta). De esta manera, promueve una oportunidad para que los alumnos hagan uso de contenidos (kichwas) minorizados y vernaculares. Además, el maestro Pedro plantea preguntas para que los estudiantes describan el afiche usando su propia variedad del castellano, el castellano amazónico peruano (Jara Yupanqui, 2012). Asimismo, propicia el uso de recursos multimodales al utilizar tanto ilustraciones como texto escrito y al emplear juegos que buscan ayudar a sus alumnos a relajarse. A través de estas prácticas, el maestro se sirve de contenidos y medios de la biliteracidad menos hegemónicos para validar los recursos que los estudiantes kichwa traen a la escuela, además de proporcionar andamiajes para el aprendizaje de la L2.

\section{Analizando e interpretando los significados sociales para los participantes}

Sin embargo, los esfuerzos del maestro Pedro relativos a un enfoque comunicativo coexisten con prácticas que limitan las oportunidades de participación de los alumnos en la tarea comunicativa. El tipo de preguntas del maestro - un claro ejemplo del patrón iniciación-respuesta-evaluación en la toma de turnos (Rymes, 2010) - le permite ser poseedor de conocimiento y autoridad que determina qué cuenta como una contribución válida, una postura consistente con el enfoque escolar tradicional en contenidos descontextualizados. De manera similar, se observa un enfoque de desarrollo centrado en el castellano productivo y oral, sin oportunidad de echar mano a prácticas lingüísticas locales, como el uso del kichwa. Ello se ve reflejado en la manera en que la participación de los alumnos se encuentra restringida por el tipo de preguntas formuladas por el maestro. Además de caracterizarse por ser preguntas que cuentan con respuestas conocidas, el maestro delimita las respuestas de los alumnos al anticipar y señalar sus respuestas (líneas 1, 3 y 9); al brindarles el artículo o la preposición que antecede al sustantivo (línea 3); y, a veces, al coincidir con sus respuestas de manera simultánea (líneas 12-13). Este patrón de preguntas posiciona a los alumnos como animadores de las respuestas esperadas por el docente (Goffman, 1979) y no como aprendices del castellano que co-construyen conocimientos a través de la interacción.

Asimismo, se debe considerar que la sesión no incluye el modelaje de las frases y expresiones comunicativas deseadas. Hacia el final, las observaciones que el maestro Pedro hace del grupo lo llevan a modificar cómo (i.e. a través de qué habilidades lingüísticas) y a través de qué medios los alumnos lograrán la tarea comunicativa. El maestro modifica la tarea original de producción oral optando por una tarea escrita y multimodal. Así, les permite a los alumnos trabajar de manera colaborativa, y usar los dibujos y el medio escrito para describir el proceso de elaborar una canoa. Aunque las prácticas letradas y escritas 114 I ciertamente pueden actuar como puentes significativos para el desarrollo de la 
producción oral, la tarea inicial que buscaba desarrollar habilidades orales y productivas en castellano no es retomada. De cierta manera, la tarea es adaptada en función de lo que el maestro Pedro percibe como dificultades de sus alumnos para producir el castellano oralmente.

\section{Describiendo una sesión del maestro Eric}

Viñeta 2: Un sajino

El maestro Eric se encuentra en el centro del aula y explica a los alumnos que hoy hablarán sobre un animal, dándoles pistas sobre este animal, dónde vive, qué come y dónde se baña. Los estudiantes rápidamente adivinan que se trata de un sajino. Eric les muestra un dibujo de un sajino, que incluye el siguiente texto en la parte inferior: “¿Qué animal es esto? Es un sajino" (Figura 3). Durante el resto de la sesión, el maestro le pide al grupo que junto a él repitan esta combinación de pregunta y respuesta, y, luego, les pide repetir la pregunta y la respuesta oralmente y sin su apoyo, de manera individual y grupal. A continuación, el maestro le pide a cada uno de sus 32 estudiantes que se paren y de manera individual les plantea la pregunta “QQué animal es esto?", la cual tienen que repetir siguiendo su ejemplo. Las respuestas de los alumnos oscilan entre "¿qué animal esto?", “qué animal es esto?” y “¿qué es animal?”. El maestro Eric repite las preguntas que no se asemejan a la suya y les pide a los alumnos repetir la frase que él modela una y otra vez, reprendiendo a los que son muy callados para que hablen con más fuerza y exhortando a los que se cubren las caras o la boca con las manos que mantengan sus brazos pegados a su cuerpo. (NC 2013-06-20) 
Figura 3

Imagen del sajino usado en clase de Eric

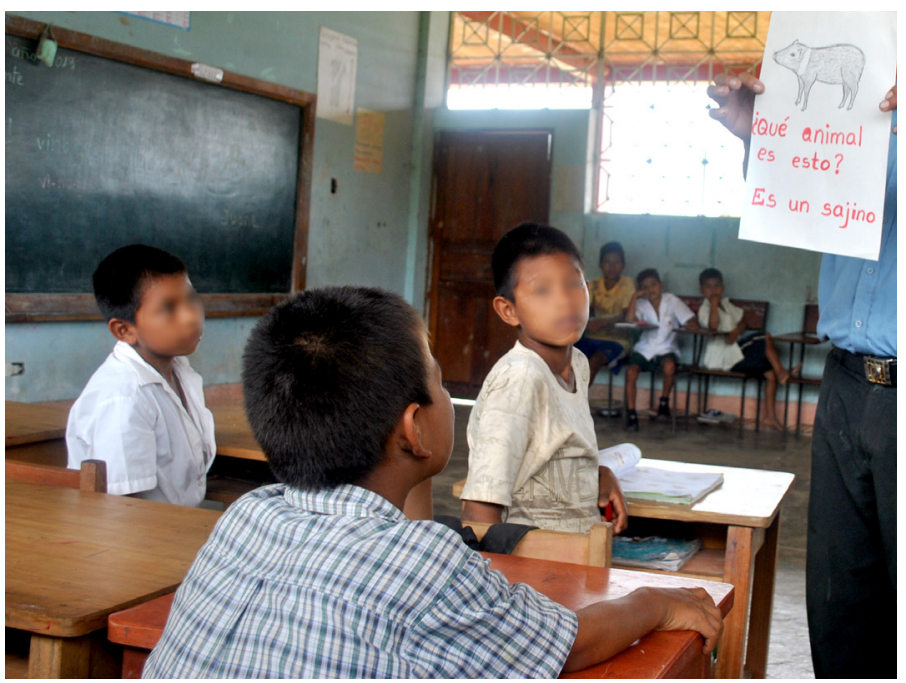

Fuente: Foto tomada por F. Kvietok Dueñas.

De manera similar a las prácticas observadas en la sesión del maestro Pedro, Eric comienza por favorecer una tarea enfocada en prácticas orales y productivas en castellano eligiendo hablar de un tema que el grupo conoce. De este modo, abre un espacio para contenidos de biliteracidad tradicionalmente menos hegemónicos. En contraste con el maestro Pedro, quien modifica la forma en que la competencia comunicativa es desarrollada en su clase, el maestro Eric no modifica la tarea comunicativa ni el medio a través del cual esta se va a lograr. En su lugar, toda la sesión es un esfuerzo continuo por superar las dificultades evidentes de sus alumnos con la tarea comunicativa y por lograr el objetivo planteado de producción oral.

\section{Analizando e interpretando los significados sociales para los participantes}

Aunque los alumnos en el grupo del maestro Eric participan de manera individual y grupal, el profesor sigue en control del habla, y genera la mayor parte del conocimiento sobre el tema tratado. Él presta mucha atención a la forma del mensaje, lo cual se refleja en su planificación de la sesión (NC 2013-06-20). Eric urge a los estudiantes a pronunciar la pregunta y la respuesta de manera completa, y con un volumen y comportamiento corporal particular. Las prácticas de corrección implican reformulaciones y correcciones más explicitas, mediante las que se le dice al grupo específicamente qué decir. Al igual que la 116 I sesión del maestro Pedro, poco o nada de kichwa oral o escrito es utilizado, lo 
cual privilegia las modalidades orales productivas y la segunda lengua en el desarrollo de la biliteracidad. En la sesión del maestro Eric, algunos de los errores producidos por sus alumnos, como el uso inapropiado o la falta de artículos o el orden de las oraciones, pueden ser ciertamente influenciados por las diferencias lingüísticas estructurales entre el kichwa y el castellano. Sin embargo, estas diferencias no son consideradas en la sesión.

Evaluando los efectos educativos para los estudiantes: Pedagogías para superar y adaptarse a la timidez percibida de los alumnos

Las prácticas de enseñanza ilustradas en las viñetas anteriores apuntan a que las maneras en las que ambos maestros dan sentido a los contextos, los contenidos, los medios, y al desarrollo en la enseñanza del castellano como segunda lengua son guiadas por ideologías de adaptación y superación a la timidez y dificultades que perciben en sus alumnos. Las ideologías de adaptación se encuentran reflejadas en las prácticas que brindan andamiajes para minimizar el fracaso de los alumnos para completar la tarea comunicativa propuesta ${ }^{9}$. La toma de turnos del maestro Pedro, que constaba de preguntas con respuestas ya conocidas, al igual que su decisión de modificar la presentación oral por una actividad de dibujo y escritura pueden ser interpretadas como prácticas de adaptación, en la medida que brindan oportunidades para que los alumnos participen o, al menos, se mantengan ocupados, lo cual evita que los estudiantes no completen las tareas planteadas o fracasen en su realización. Sin embargo, no brinda oportunidades significativas para el desarrollo oral del castellano, lo cual es el propósito de la actividad y de la clase de castellano. Las prácticas de adaptación, asimismo, incluían el uso de juegos lingüísticos y canciones con poca conexión con el tema de la sesión, y los estudiantes mostraban poca comprensión de lo que cantaban o de las letras de las canciones (NC 2013-06-19). A pesar de que los juegos lingüísticos y las canciones pueden ser utilizados de manera provechosa para apoyar el aprendizaje de una segunda lengua y no necesariamente reflejan prácticas de adaptación, este fue el uso que mayormente se observó.

En contraste, un enfoque que busca superar la supuesta timidez o las dificultades de los alumnos no está principalmente enfocado en permitir que los estudiantes logren sobrellevar las sesiones, sino que los empuja a que logren la tarea en cuestión o produzcan el lenguaje deseado. El uso de prácticas o ejercicios de repetición que formaban parte de la sesión del maestro Eric ejemplifica este enfoque. Las prácticas de enseñanza que buscaban superar las dificultades percibidas de los alumnos también contaban con un fuerte enfoque en la forma del mensaje - tanto la forma lingüística como el comportamiento corporal asociado con la realización exitosa de una tarea-. Por ejemplo, gran parte de las observaciones de Eric se centraban en corregir el volumen de la voz y la

9. Al respecto, se puede consultar Hornberger y Chick (2001) sobre "safe-talk". 
postura de sus alumnos, sin ningún o muy poco énfasis en la comprensión del mensaje de sus estudiantes.

Las prácticas con un enfoque en la superación y la adaptación de la supuesta timidez y las dificultades percibidas en los estudiantes también pueden coexistir en una misma sesión. En una sesión de segundo grado del maestro Daniel, los alumnos comenzaron participando en diálogos introductorios, acompañados por prácticas de repetición. La sesión comenzó con el maestro Daniel modelando la frase “¿Cómo se llama tu papá o tu mamá??". Sin embargo, cuando fue el turno de la primera pareja de estudiantes de repetir esta misma frase frente al salón, se preguntaron lo siguiente “¿Cómo te llamas?”. El maestro Daniel no intervino y permitió que el diálogo entre la pareja continúe (NC 2013-07-02).

De esta manera, la misma sesión reflejó ideologías tanto de superación, al ofrecer un formato de participación de repetición familiar para los estudiantes, y de adaptación, al dejar de lado la frase que se buscaba desarrollar por otra ya conocida y practicada múltiples veces. Dado que las presentaciones personales y los saludos eran el enfoque constante de las sesiones del primer, segundo y tercer grado, las actividades que volvían a revisar frases relacionadas con estas prácticas permitían a los alumnos realizar las tareas planteadas, aunque sin proveerles de suficientes oportunidades para expandir sus habilidades de producción y comprensión oral en su L2. En esa medida, aunque las prácticas comunicativas inspiradas en ideologías de superación y adaptación pueden hacer uso de contenidos familiares para los estudiantes y de medios visuales que complementan a las interacciones orales y escritas, estas prácticas finalmente menoscaban el desarrollo de la biliteracidad en otras maneras.

A continuación, consideramos el potencial del monitoreo etnográfico colaborativo informado por las continuidades de biliteracidad para apoyar a los docentes a reflexionar críticamente sobre sus prácticas comunicativas del aula.

\section{Escala 3: Construyendo pedagogías de la voz}

Vemos al monitor etnográfico como un actor social que puede involucrarse en influir en las desigualdades lingüísticas. Una de las fortalezas del monitoreo etnográfico es que su método no es predeterminado, sino que se afina al contexto local (Hymes, 1980). En el caso del Alto Napo, el involucramiento tomó la forma de colaboración con los maestros para desarrollar lo que aquí llamamos "pedagogías de la voz", es decir, pedagogías que crean condiciones para que la voz estudiantil indígena se desarrolle y florezca (Hornberger, 2006). Sugerimos que un modo de realizar esta pedagogía es apoyar a los estudiantes en la negociación de los múltiples puntos de las continuidades de la biliteracidad mientras desarrollan sus habilidades comunicativas en la segunda lengua.

La colaboración entre la monitora y los maestros surgió durante un perío-

118 I do de dos meses e implicó una negociación continua de sus roles y las estructu- 
ras participativas. Durante el primer taller para maestros que Kvietok Dueñas realizó, dirigió una serie de actividades con un enfoque en la práctica pedagógica investigativa. Al concluir el taller, se sintió decepcionada por no haber logrado el nivel de autorreflexión que esperaba. Tampoco, observó que los maestros aplicaran algunas de las prácticas pedagógicas que habían discutido en sus aulas (NC 2013-06-19). Con el tiempo, comenzó a darse cuenta de que sus nociones de participación y las de los maestros diferían. Mientras que, para ella, su rol como monitora era escuchar y dialogar con los maestros acerca de sus prácticas de enseñanza de la segunda lengua, los maestros esperaban, más bien, que ella les demostrara algunas de estas prácticas. Este hallazgo no fue un proceso lineal ni inmediato, sino emergente, en el curso de las entrevistas y observaciones de aula que realizaba. Gradualmente, Kvietok Dueñas asumió roles más activos, no solo en respuesta a los pedidos de los maestros, sino también debido a su creciente entendimiento de las experiencias de los maestros con el proceso de acompañamiento y a su propia reflexión sobre cómo modelar prácticas sin impedir a los maestros que desarrollen sus propias didácticas.

Para ilustrar cómo aplicamos la noción de las pedagogías de la voz, describimos una clase modelo del castellano como L2, y la analizamos e interpretamos empleando las continuidades de la biliteracidad. Luego, recurrimos a las conversaciones y reflexiones sobre planificación y enseñanza que sostuvieron Kvietok Dueñas y los maestros para evaluar la construcción de pedagogías de la voz. Puesto que fue un proyecto breve, el monitoreo etnográfico no incluyó un seguimiento posterior a las aulas de los maestros.

\section{Describiendo la clase modelo}

Viñeta 3: Compra y venta con comerciantes del río

Basándose en observaciones etnográficas del uso del castellano en el Alto Napo y en conversaciones con estudiantes, Kvietok Dueñas planificó una sesión modelo con el tema de las transacciones con comerciantes del río, la cual implementó en el cuarto, quinto y sexto grado en colaboración con los maestros. En la sesión modelo, como paso previo a los diálogos de compra y venta, los estudiantes generaban una lista de artículos de venta, dividiéndolos en dos grupos de acuerdo con el determinante masculino o femenino que llevaban, e. g. un cartucho (m.) o una linterna (f.). Esta actividad provocaba una reflexión, guiada por la monitora y el maestro, sobre las diferencias entre las lenguas, dada la existencia de determinantes en castellano, figura que no existe en kichwa. Preguntas como “ ¿Observaron diferencias entre el castellano y el kichwa?”, “¿Es importante utilizar estos artículos en castellano?”, “ipor qué sí/no?” y “QQué estrategias podemos utilizar para aprenderlos?” enmarcaron la sesión. 
Luego, los estudiantes realizaron un juego de roles, en escenas de compra y venta con comerciantes del río de varios artículos que habían generado a partir de su propio conocimiento de los encuentros, haciendo uso del guion de diálogo escrito y ensayado por la monitora, o desarrollando sus propios diálogos. De acuerdo con el grado escolar, existían diferencias de duración y complejidad del diálogo, como también de las actividades de andamiaje y refuerzo. Durante las clases modelo, Kvietok Dueñas y los maestros incluían actividades en pares y también oportunidades para participar en ambas lenguas. Las discusiones en grupos pequeños facilitaban que los estudiantes se escucharan y comunicaran entre sí, tomando diferentes roles que implicaban destrezas comunicativas tanto de comprensión como de expresión. Al final, cuando los estudiantes reportaban sus trabajos a la clase entera, se enfatizaba la participación oral con apoyo (opcional) de material escrito.

\section{Analizando e interpretando los significados y patrones emergentes para los maestros}

Las pedagogías de la voz descritas incluyen estrategias que ejercen las capacidades de los alumnos a lo largo de cada una de las continuidades de biliteracidad, y que visibilizan y rompen el desbalance hegemónico del poder que favorece un extremo de cada continuidad sobre otro. Por ejemplo, en cuanto a los contenidos y los contextos de la biliteracidad, el intento de atender a la voz estudiantil implicaba que la monitora y los maestros consideren qué situaciones y contenidos serían lo más propicios para el desarrollo de destrezas productivas orales en la lengua dominante. En la clase modelo, los alumnos habían aprovechado la actividad conocida de la compra y venta con comerciantes del río y sus prácticas comunicativas relevantes. Además de eso, habiendo antes reflexionado sobre cómo el desarrollo de la biliteracidad puede sufrir aún cuando se incluye contenido local y medios visuales, los maestros y Kvietok Dueñas emplearon actividades que apuntaban hacia el empleo de estructuras participativas (Philips, 1972) más interactivas, apoyando a las continuidades L1-L2, orales-escritas, de comprensión y expresión en el desarrollo de la biliteracidad. Así, en la clase modelo, se veía un cambio hacia estructuras participativas que colocaban a los estudiantes en roles de oyentes, participantes y creadores expertos de saberes. Asimismo, la clase ofrecía andamiajes para el aprendizaje de ciertas frases específicas en la L2, tanto en forma oral como escrita. En materia de las continuidades de los medios de biliteracidad, se buscaba provocar una reflexión metalingüística en la clase sobre el kichwa y el castellano: se preguntaba explícitamente acerca de diferencias y similitudes entre las lenguas, y se fomentaba la reflexión sobre el aprendizaje de lenguas. 


\section{Evaluando la clase modelo en colaboración con los maestros kichwas}

La clase modelo conducía a diálogos entre Kvietok Dueñas y los maestros sobre posibles escenarios de futuras pedagogías de la voz en sus contextos. En este punto, planteamos una breve reflexión y evaluación: ¿en qué medida se podría decir que la clase modelo conducía a los maestros hacia nuevos entendimientos?

En relación con los contextos y los contenidos de la biliteracidad: en las sesiones y talleres subsecuentes a la clase modelo, los maestros comentaban a menudo que las múltiples situaciones de interacción comunicativa diaria podrían servir de materia para clases futuras, incluido el uso del castellano por los alumnos cuando jugaban fútbol y vóleibol, así como el uso del castellano en asambleas comunales, la radio y visitas a la posta médica. Las actividades del monitoreo propician que los maestros exploren estilos comunicativos típicos de la vida diaria de los alumnos para el desarrollo de la biliteracidad, que consideren las audiencias y los propósitos comunicativos que son significativos para ellos y sus alumnos, y que tomen en cuenta cómo esto se relaciona con los usos de la lengua dominante en la comunidad y con sus objetivos de aprendizaje de lenguas.

La concientización crítica del lenguaje puede apoyar a que maestros y alumnos reflexionen sobre las ideologías subyacentes a sus propias prácticas comunicativas y las de la comunidad, y así conducir a su mayor entendimiento de cómo es que "las prácticas comunicativas contribuyen al mantenimiento o la resistencia de representaciones particulares del mundo y de las relaciones del poder" (Clark y Ivanic, 1999, p. 67). La postura de una conciencia crítica en las actividades del monitoreo creó oportunidades en las que los maestros consideraron instancias de discriminación lingüística, contextos contenciosos del uso del castellano en las comunidades kichwa, y las ventajas y desventajas del bilingüismo. En los talleres, los maestros formulaban preguntas guía para diálogos futuros en sus aulas: ¿cómo interactuamos nosotros/nuestras familias con los comerciantes del río?, ¿cuales son algunos retos que enfrentamos?, ¿qué quisiéramos cambiar?, ¿por qué y para qué son importantes el castellano y el kichwa en nuestras comunidades y afuera?, ¿cuándo y cómo fue el inicio de la educación bilingüe kichwa?

En relación con el desarrollo y los medios de la biliteracidad: otro resultado de nuestra evaluación colaborativa con los maestros fue su mayor interés en implementar estrategias participativas enfocadas en el alumno, no solo en la clase de castellano sino a través de todo el currículo, extendiendo las posibilidades para el desarrollo de la biliteracidad en toda la escolaridad. Otras estrategias incluyeron el reemplazo del énfasis en frases sueltas por el uso de diálogos significativos, y la planificación de una secuencia de clases de castellano L2 en vez de clases sueltas. Otra observación compartida entre la monitora y los maestros fue la necesidad de ir más allá de planificar las clases del castellano L2 grado por grado, y, en cambio, planificar de acuerdo con los diferentes niveles del desarrollo del castellano, debido a que existen múltiples niveles dentro del mismo grado. 
Los maestros y Kvietok Dueñas también tomaron en cuenta cómo comentarios referentes a la supuesta timidez de los alumnos y sus dificultades permanentes con el castellano, y las reprimendas verbales podrían transformarse en diálogos constructivos acerca del aprendizaje de lenguas. Juntos generaron preguntas como las siguientes: ¿cómo aprendemos?, ¿qué nos hace sentir confianza o ansiedad al aprender el castellano?, ¿cómo aprendieron el castellano nuestros maestros y padres? Esta reflexión metalingüística abrió oportunidades para que los docentes compartieran sus trayectorias de desarrollo de la biliteracidad. Este diálogo subrayó la diversidad de sus trayectorias y los alentó a considerar cómo podrían movilizar y utilizar esa diversidad en sus aulas como vehículo de reflexión sobre procesos de aprendizaje del castellano.

El mapeo de la enseñanza de la biliteracidad en colaboración con los maestros puede apoyar a evaluar y desarrollar prácticas pedagógicas localmente enraizadas que abran oportunidades para que los alumnos se expresen y sean escuchados en sus aulas actuales, así como en los muchos contextos que navegan y navegarán en el futuro. Emplear pedagogías de la voz en la enseñanza de biliteracidad en contextos poscoloniales de la educación bilingüe no implica simplemente de la lengua de instrucción —en este caso, el castellano o la lengua indígena-, sino abordar las jerarquías de poder y las ideologías lingüísticas locales, al mismo tiempo que se abordan las aspiraciones y los propósitos locales del bilingüismo.

\section{Implicancias: Mapeando y reencaminando la enseñanza de biliteracidad hacia las pedagogías de la voz en contextos indígenas}

Nuestro mapeo de la enseñanza de biliteracidad en ciclos iterativos del monitoreo etnográfico hymesiano -atravesando las ecologías locales de desigualdad lingüística, las prácticas comunicativas del aula, y la creación de pedagogías de la voz - ha iluminado cómo los maestros bilingües kichwas negociaron las continuidades de la biliteracidad a medida que transformaban sus objetivos de enseñanza de castellano como segunda lengua en prácticas en el aula. En nuestro mapeo de las ecologías lingüísticas del Alto Napo, vimos que las prácticas educativas y comunicativas en kichwa y en castellano se encontraban enmarcadas dentro de contextos de colonialidad y resistencia por las experiencias e ideologías de aprendizaje de lenguas de los maestros, y por metas más amplias de representación política y bienestar socioeconómico para las comunidades kichwa.

Mapeando las prácticas comunicativas del aula, exploramos en qué medida el énfasis que ponían los maestros en diferentes puntos de las continuidades de biliteracidad en sus clases apoyaba los objetivos de desarrollo de capacidades productivas orales en castellano en sus alumnos y así cumplían las necesidades comunicativas comunitarias. También, consideramos hasta qué punto sus 122 I pedagogías inspiradas en ideologías de superación y adaptación a la supuesta 
timidez o falta de motivación de sus alumnos, paradójicamente, podían tener el efecto inesperado y no deseado de mantener en vez de contrarrestar la visión de los alumnos como aprendices del castellano con dificultades continuas. Si bien es cierto que el desarrollo de capacidades comunicativas orales en castellano de los alumnos estaba en proceso, que ellos experimentaban dificultades en este proceso, y que encontraban muy poco refuerzo para el castellano fuera del colegio, queremos recalcar que las pedagogías de sus maestros también podían contribuir a las dificultades a las que se enfrentaban permanentemente. En nuestro mapeo de la implementación y evaluación de una clase modelo, señalamos cómo las prácticas comunicativas del aula pueden ser reencaminadas hacia un mejor aprendizaje para los alumnos.

El mapeo etnográfico no es un proceso lineal ni carece de desafíos. Hemos tratado de ilustrar y enfatizar los procesos y significados iterativos, cíclicos, multinivelados, emergentes y cambiantes del monitoreo, mapeo, y colaboración. Asimismo, hemos buscado abogar por la importancia y necesidad de una reflexión continua a lo largo del proceso. Una comprensión acumulativa -en torno a cómo los roles son negociados por los monitores y los participantes, cómo las diferentes estructuras de participación pueden cerrar o abrir vías de colaboración, y cómo las diversas dinámicas pueden incluir o excluir a actores y significados - enriquece nuestro empeño colectivo de mapear y reencaminar la enseñanza de biliteracidad hacia la justicia educativa en contextos indígenas.

Existen tensiones y desigualdades significativas y profundamente arraigadas en la enseñanza de lenguas mayoritarias a alumnos indígenas en contextos poscoloniales. Mapear la enseñanza con el monitoreo etnográfico a través de las continuidades de biliteracidad, sugerimos, es una manera prometedora de avanzar hacia la creación de pedagogías de la voz que apoyen a los alumnos minorizados en su aprendizaje de lenguas mayorizadas.

\section{Agradecimientos}

En primer lugar, queremos agradecer a los maestros kichwas de la red Cebes del Alto Napo por su generoso compartir, tanto de su práctica docente como de su tiempo. Asimismo, agradecemos el apoyo del equipo de Formabiap en Iquitos para facilitar esta investigación. Estamos agradecidas con la audiencia del VI Simposio Internacional de Bilingüismo y Educación Bilingüe de Latinoamérica realizado en Lima, Perú, donde presentamos este trabajo por primera vez. Reconocemos el apoyo del Graduate School of Education Dean's Summer Research Funding, que financió el trabajo de campo de nuestro estudio. Finalmente, apreciamos mucho y agradecemos el apoyo del comité de esta revista y de las editoras de Anthropology and Education Quarterly para publicar este trabajo en su versión en castellano. 


\section{Referencias bibliográficas}

Aidesep (2013). Organizaciones Kichwas del Napo emiten pronunciamiento y reclaman inicio de procesos de consulta. Aidesep.

Aikman, S. (1999). Intercultural Education and Literacy: An Ethnographic Study of Indigenous Knowledge and Learning in the Peruvian Amazon. Philadelphia: John Benjamins.

Ashanga Jota, G., Vera, R. E., San Román, G. y Tushupe, L. (1990). Catorce años de educación bilingüe en la Amazonía: Análisis y perspectiva. Amazonía Peruana, 10(18), 53-66.

Clark, R. y Ivanic, R. (1999). Editorial. Raising critical awareness of language: A curriculum aim for the New Millennium. Language Awareness, 8(2), 63-70.

De Korne, H. y Hornberger, N. H. (2017). Countering unequal multilingualism through ethnographic monitoring. En M. Martin-Jones y D. Martin (Eds.), Researching multilingualism: Critical and ethnographic approaches (pp. 247-58). New York: Routledge.

Goffman, E. (1979). Footing. Semiotica, 25, 1-29.

Gumperz, J. J. (1964). Linguistic and social interaction in two communities. American Anthropologist, 66(6, II), 137-153.

Hornberger, N. H. (1989). Continua of biliteracy. Review of Educational Research, 59(3), 271-96.

. (1990). Creating successful learning contexts for bilingual literacy. Teachers College Record, 92(2), 212-29.

. (2002). Multilingual language policies and the continua of biliteracy: An ecological approach. Language Policy, 1(1), 27-51.

. (2003). Continua of biliteracy: An ecological framework for educational policy, research and practice in multilingual settings. Clevedon, UK: Multilingual Matters.

Hornberger, N. H. (2006). Voice and biliteracy in indigenous language revitalization: Contentious educational practices in Quechua, Guarani, and Māori contexts." Journal of Language, Identity, and Education, 5(4), $277-92$.

. (2009). Hymes's linguistics and ethnography in education.

Text \& Talk: An Interdisciplinary Journal of Language, Discourse \& Communication Studies, 29(3), 347-58.

.(2014). On not taking language inequality for granted: Hymesian traces in ethnographic monitoring of South Africa's multilingual language policy. Multilingua, 33(5/6), 625-47.

Hornberger, N. H. y Chick, J. K. (2001). Co-constructing school safetime: Safetalk practices in Peruvian and South African classrooms. En: M. Heller 
y M. Martin-Jones (Eds.), Voices of authority: Education and linguistic difference (pp. 31-55). Westport, CT: Ablex Publishing.

Hornberger, N. H. y Skilton-Sylvester, E. (2000). Revisiting the continua of biliteracy: International and critical perspectives. [Fourteen years of bilingual education in the Amazon: Analysis and prospects.] Language and Education: An International Journal, 14(2), 96-122.

Hymes, D. H. (1980). Language in education: Ethnolinguistic essays. Washington, DC: Center for Applied Linguistics.

Hymes, D. H. (Ed.) (1981). Ethnographic monitoring of children's acquisition of reading/language arts skills in and out of the classroom: Final Report to the National Institute of Education, Philadelphia. Recuperado de la base de datos ERIC (ED208096).

Jara Yupanqui, I. (2012). Peruvian Amazonian Spanish: Linguistic variation, language ideologies and identities. Sociolinguistic Studies, 6(3), 445-469.

Jung, I. y López, L. E. (eds.) (2003). Abriendo la escuela: Lingüística aplicada a la enseñanza de idiomas. Madrid: Ediciones Morata.

Kvietok Dueñas, F. (2015). Negotiating ideological and implementational spaces for Indigenous languages in Peru. Working Papers in Educational Linguistics, 30(1), 21-41.

Medina, D. (2009). Construyendo comunidades y escuelas para el bien estar Cebes. KÚÚMU, 8, 18-21.

Mercier, J. M. (1983). El Kichwa del Napo. En A. Corbera (Ed.), Educación y lingüística en la Amazonía peruana (pp. 28-64). Lima: CAAAP.

Philips, S. (1972). Participant Structures and Communicative Competence: Warm Springs Children in Community and Classroom". In C. Cazden, V. John and D. Hymes (eds.), Functions of Language in the Classroom (pp. 370-394). New York: Teachers College Press.

Quijano, A. (2000). Coloniality of power, eurocentrism and Latin America. Nepantla: Views from the South, 1(3), 533-580.

Ruiz, R. (1997). The empowerment of language-minority students. En A. Darder, R. D. Torres and H. Gutiérrez (Eds.), Latinos and education: A critical reader (pp. 319-328). New York, NY: Routledge.

Rymes, B. (2010). Classroom discourse analysis: A focus on communicative repertoires. En N. H. Hornberger y S. L. McKay (Eds.), Sociolinguistics and Language Education (pp. 528-546). Bristol, UK: Multilingual Matters.

Trapnell, L. (2010). Informe Final de Consultoría Evaluación Intermedia del Proyecto: "Construyendo una propuesta pedagógica para los pueblos indígenas amazónicos (2008-2011)". Documento interno, Formabiap.

Van der Aa, J. y Blommaert, J. (2011). Ethnographic monitoring: Hymes's unfinished business in educational research. Anthropology and Education Quarterly, 42(4), 319-334. 